\title{
BricksAR: exploring the potential of augmented reality to inform assembly processes in construction
}

\author{
João Pedro Andrade Caixeta \\ Faculdade de Arquitetura, urbanismo e Design \\ (FAUeD) \\ Universidade Federal de Uberlândia \\ (UFU) \\ Uberlândia, Brasil \\ jpedrocxta@gmail.com
}

\author{
André Luís de Araujo \\ Faculdade de Arquitetura, urbanismo e Design \\ (FAUeD) \\ Universidade Federal de Uberlândia \\ (UFU) \\ Uberlândia, Brasil \\ andre.araujo@ufu.br
}

\begin{abstract}
The use of Augmented Reality (AR) systems in construction processes can represent an essential transformation in the communication between design and production. However, supposing that design-production translations can be obtained from several manufacturing methods (such as robotic, manual, modular, non-modular, and others), there are not enough studies that explored the potential uses of AR as an assistant for assembly operations. To tackle this problem, this ongoing research proposes to investigate the potentials of a low-cost and marker-based AR system to conduct different manual assembly processes. With the observational focus on aspects of precision and feasibility, we used scientific reductions based on modeling, simulation, and prototyping to provide inferences about the proposed tool's behavior in the real world.
\end{abstract}

Keywords-Augmented reality, Digital construction, Complex shapes, Assembly process

\section{INTRODUCTION}

The use of computing as a tool to drive construction processes is not recent and has some remarkable points in history. For instance, Pierre Bézier's initiatives at Renault plants in the 1960s sought to replace automotive manufacturing standards from formerly hand-tooled fabrication processes to automated numerical controlled ones, consequently solving several assembly problems [1]. Since then, many pieces of research have attempted to improve relations between design and manufacturing using different person-machine communication protocols [2]. Nowadays, Computer-Aided Design (CAD) and Computer-Aided Manufacturing (CAM) is an essential part of most industries in the field of Architecture, Engineering, Construction, and Operation (AECO).

The introduction of CAD and CAM technologies profoundly redefined the nature of what is designed and built by removing Euclidean geometry restrictions imposed by traditional design and manufacturing techniques [3]. Conventional fabrication methods used by this sector include computer numerically controlled (CNC) systems, such as cutting (laser, waterjet or plasma cutting), subtractive (milling), additive (3D printing), and formative (numerically controlled bending) fabrication [4]. Concrete 3D printing [5], use of robotic arms to assemble knotty modular walls [6], and the use of drone swarms to build freeform shapes [7][8], are some of the recent proposals for new computer-controlled construction methods.

Although literature regularly reports novel digital manufacturing processes, such methods usually require extremely qualified labor and expensive equipment, preventing efficient implementation strategies, especially in non-developed countries. Additionally, general digital fabrication processes often lack the human intuition aspect in material production [9]. In order to tackle these problems, some applications have explored the potential of augmented reality (AR) use as an essential communication tool between design and production [10].

However, even with these studies in the literature, some nuances about AR's applications in fabrication remain obscure. One of them would be its use as an assistant for the assembly of complex shapes, supposing that these can be obtained from several manufacturing methods (such as robotic, manual, modular, non-modular, and others). Based on the assumption that AR's use in these processes presents different potentialities, this research proposes investigating them from comparative experiments. A low-cost and markerbased AR system's potentials were investigated from a prospective point of view about manual assembly processes. With the observational focus on aspects of precision and feasibility, we used scientific reductions based on modeling, simulation, and prototyping to propose inferences about the proposed tool's behavior in the real world.

In the next section of this paper, we briefly review current AR technology applications in the AECO sector. Then, we detail the method used in the experiments. Next, we discuss preliminary outcomes and conclude the paper explaining future steps.

\section{AR APPLICATIONS IN AECO}

The integration of AR visualization with other computer vision techniques has been used as a critical detection tool in different scenarios. For instance, applications of this type allowed the identification of potential hazards and accessibility issues in indoor environments by measuring the space between furniture [11]. Construction safety analysis also represents an emerging use of this technology. Research indicates the potential of AR systems to identify hazards and conduct safety inspections at construction sites [12]. In outdoor applications, AR has been used to measure the number of green areas in a given landscape, allowing the conclusion on the necessity of local/urban interventions on the green space [13].

The use of AR as a modeling tool in the design process in order to improve aspects of cognition between designer and form is another application explored in the reviewed literature. AR opens the possibility to visualize a shape inserted in the real environment and intuitively manipulate it through 3D transformations [14]. In addition to a graphical return, this type of application allows users to manipulate a shape by 
touching real objects while understanding the geometric implications of the changes made on virtual ones [15]. In the AECO area, this can represent a more consistent communication between design and construction, regardless of the construction method used, also supporting learning about the assembly procedures [16].

Another substantial part of the recent applications that use AR in AECO is to supervise, collaborate, and control digital fabrication systems. In some of these experiments, computer instructions allowed users to evaluate the production trajectories of the equipment, employing subtractive processes such as CNC milling machines and 3D printing [17] additive processes [18]. The overlap between virtual and real objects enables the problems anticipation during the manufacturing process from constant feedback [19] [20]. This access to production conditions via AR creates a more interactive fabrication workflow, allowing the designer to apply changes in the manufacturing process and instantly perceiving the effects of this change [21].

Unlike production through digital fabrication, construction processes generally demand bi-dimensional instances of information, and, in this sense, AR allows establishing fast correlations between the plant and 3D models [22]. However, the integration of holographic design, fabrication, and assembly could turn obsolete the use of 2D documentation to instruct buildings production [23]. Furthermore, AR could open a different paradigm from an ecological concept of design, once it puts the designers close to the crafts during the design process [24]. This technology also enables partial interventions and experimentation, once the virtual graphic returns can be integrated with real parts already consolidated [25]. It is possible to confirm that AR allows different levels of intervention in the assembly process, this technology has proven gains when used to guide assembly of objects with greater geometric complexity.

\section{METHODS}

In this research, we set up experimental and simulation scientific reductions to analyze the efficiency of a markerbased AR system in conducting manual assembly processes. We conjecture about possible advances in manufacturing operations based on the assumption that augmented reality alters the essence of the investigated assembling tasks from inductive logic. Seeking to interpret this experiment's meanings in a broader context, we establish a comparison between two models assembly process with the same treatment to define inferences about the particularities of AR guided assembly in different contexts. The prototypes assembly experiments refer to two situations: 1) assembly of knotty walls using bricks of standardized geometry (BricksAR); 2) assembly of complex bar structures using standardized geometry connections (NodesAR). This paper presents a work-in-progress about BricksAR development.

We designed the virtual models in Rhinoceros 3D modeling software and Grasshopper diagrammatic scripts plugin (Robert McNeel \& Associates). The wavy wall shape generation used meta-objects - implicit surfaces obtained by mathematical formulas that use the information contained in previous objects to generate a new one with organic and fluid final appearances - algorithms that produced a freeform, complex and modular wall. The obtained geometry was then optimized to guarantee that all blocks had a minimum area of contact with the lower layer, thus ensuring the feasibility of the prototype. The final model was $26 \mathrm{~cm}$ in diameter, had seven layers and a total of 244 blocks (Figure 1).

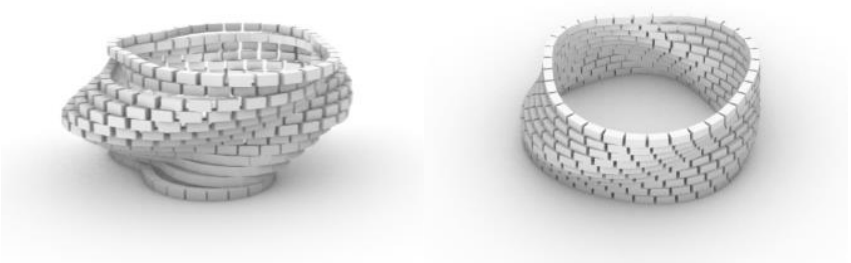

Figure 1 - The model generated by the algorithm (left); the same model after the optimization process (right). Source: Authors.

BricksAR experiment used a simple AR marker system that captures and recognizes a marker position and then displayed the model's assembly instructions overlapping the live camera footage on a bi-dimensional screen (Figure 2). To recognize the marker and display assembly instructions, we built a piece of software using Unity game engine (Unity Technologies) and Vuforia (PTC Inc.) software development kit (SDK) for Unity (Figure 3). Vuforia SDK was selected due to its ability to recognize fixed markers accurately. The scripts that control model's assembly phases sequencing were written using C\# programming language in Visual Studio IDE (Microsoft Inc.).

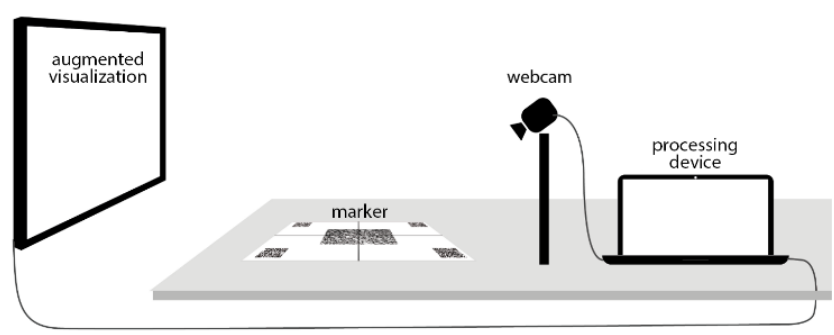

Figure 2 - System's arrangement used in this experiment. Source: Authors.

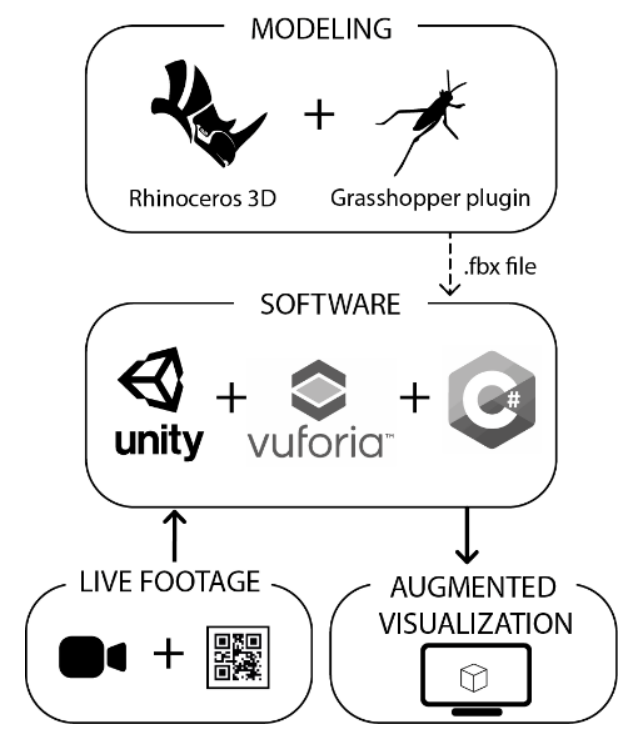

Figure 3 - An overview of the tools used. Source: Authors.

The proposed system generates an augmented visualization displayed on a large monitor (Figure 2) to support multi-user collaboration. This AR visualization guides the assembly process by indicating where each block should be manually positioned and highlighting intersection 
areas with lower layer blocks, indicating where glue should be applied (Figure 4). Interaction with the virtual model occurs using the processing device (laptop) keyboard. The users can switch between each of the seven layers exhibition passed to the monitor.

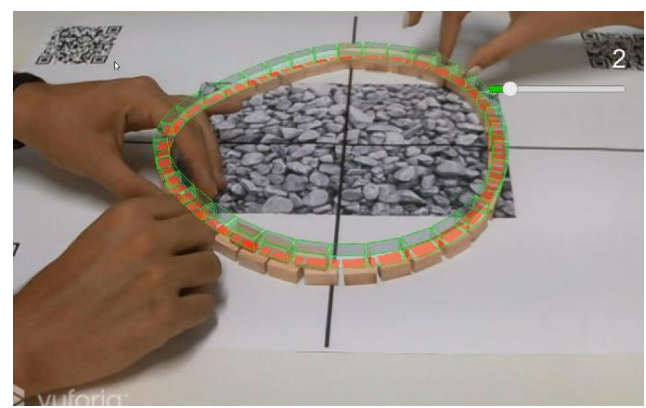

Figure 4 - Virtual model overlapping real marker; orange areas indicates glue application regions; the top-right number indicates the current layer displayed. Source: Authors.

\section{PRELIMINARY OUTCOMES AND DISCUSSION}

This ongoing research aims to compare two different ARguided assembly methods: a complex, modular layered one and a complex, non-modular spatial one. In this paper, we discuss the proposed tool efficiency by analyzing aspects of precision and feasibility related to the first concluded experiment only (Figure 5). Using scientific reductions, we also present some inferences about the behavior of this tool in different scenarios.

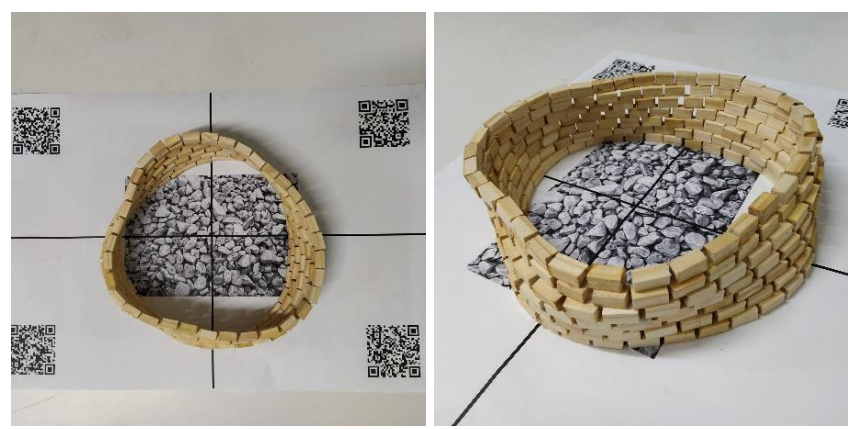

Figure 5 - prototypes assembled with AR assistance. Source: Authors.

BricksAR prototype manufacturing process took approximately $1 \mathrm{~h} 40 \mathrm{~min}$ and was carried out by two people working collaboratively: a time-lapse of the process is available on the web [26]. In terms of construction accuracy, the small size of the blocks and their irregularities proved to be challenging. Height differences between the various pieces was also a significant problem in assembling due to the consequent irregularity in the upper layers, thus preventing the correct positioning of new blocks. Also, the marker recognition system encountered difficulties with perspective deformations caused by fixed camera positioning. Another challenge encountered was that the process of positioning each block manually commonly occluded the marker from the camera capture, causing the augmented objects to flicker on the screen.

The ultimate precision of the prototype was somewhat limited due to the challenges outlined above. The full model's appearance was visually inspected, and it was possible to recognize orientation and positioning errors, especially at the upper layers. However, the assembly process findings for this experiment cannot be generalized for this tool's application on larger scales. Considering that manual accuracy is minimal at smaller scales [27], by increasing the model size and guaranteeing the regularity of the modular components, we can assume that overall precision would also increase. Problems regarding hand occlusion and perspective distortions could be solved by using head-mounted displays (HMDs) instead of a fixed camera. By providing the users the ability to visualize the object's assembly instructions from multiple views, accurate positioning becomes more intuitive.

Despite experienced issues in the experiment, the obtained results allow us to confirm the potential of AR technologies in construction and fabrication. Such a prototype would be impractical if traditional techniques using blueprints to indicate the position and angle of each of the 344 blocks were used. Another perceived benefit of this method is the possibility of collaborative work in the process. Due to the reduced scale of the model, two people worked simultaneously to complete the assembly task. However, more workers could be involved in the process if prototype dimensions were favorable.

Furthermore, the proposed tool can serve as a bridge between digital manufacturing and manual manufacturing, thus creating a more humanized and inexpensive path from design to production. The use of this method can also provide designers and architects experimentation freedom to new forms and fabrication approaches and potentially democratize construction processes by allowing the use of unskilled labor.

\section{CONCLUSION AND FUTURE STEPS}

This research investigated the potentials of a contemporary assembly technique for complex shaped geometries based on a simple, low-cost, and marker-based AR system. Preliminary outcomes suggest that the use of this tool for small scale prototypes presents precision limits. However, we recognized several benefits from the proposed experiment. Collaborative potential, cost efficiency, construction processes democratization, and design experimentation freedom encouragement are some of the potentialities of this digitally enhanced assembly method. In the future, we intend to carry on the process of perfecting the project by studying AR efficiency in guiding the construction of more towering prototypes. From the next experiments, we expect to provide insights into the tool's effectiveness not only for the execution of reduced models but also for full-scale constructions.

\section{ACKNOWLEDGMENT}

The authors thankfully acknowledge the financial support of the Brazilian National Council for Scientific and Technological Development (CNPq).

\section{REFERENCES}

[1] P. Laurent, and P. Sablonnière, "Pierre Bezièr: an engineering and a mathematician," Journal of Computer Aided Geometric Design, vol. 18, pp. 609-617, September 2001

[2] F. Gramazio, and M. Kohler, Digital Materiality in Architecture. Baden: Lars Muller, 2008.

[3] W. Mitchell, M. Mccullough, Digital Design Media. New Jersey: John Wiley \& Sons, 1995.

[4] B. Kolarevic, Architecture in the Digital Age: design and manufacturing. London: Routledge, 2003.

[5] C. Gosselin, R. Duballet, P. Roux, N. Gaudilliere, J. Dirrenberger, P. Morel, "Large-scale 3D printing of ultra-high performance concrete - 
a new processing route for architects and builders," Materials \& Design, vol. 100, pp. 102-109, March 2016.

[6] F. Gramazio, and M. Kohler. The Robotic Touch: how robots change architecture. London: Park Publishing, 2014.

[7] J. Willmann, M. Knauss, T. Bonwetsch, A. Apolinarska, F. Gramazio, and M. Kohler, "Robotic timber construction: expanding additive fabrication to new dimensions," Automation in Construction, vol. 61, pp. 16-23, January 2016.

[8] F. Augugliaro, S. Lupashin, M. Hamer, C. Male, M. Hehn, M. Mueller, J. Willmann, F. Gramazio, M. Kohler, R. D'Andrea, "The flight assembled architecture installation: Cooperative construction with flying machines," IEEE Control Systems Magazine, vol. 34, pp. 4664, August 2014.

[9] R. Johns, "Augmented Materiality: modelling with material indeterminacy," in: Fabricate 2014: negotiating design \& making, F. Gramazio and M. Kooler, Eds. London: UCL Press, 2017, pp. 216 223.

[10] Qualcomm. Augmented and virtual reality: the first wave of 5G killer apps. New York: ABI Research, 2017. Available in: https://www.qualcomm.com/

[11] S. Eloy, L. Dias, L. Ourique, M. Dias, "Home Mobility Hazards Detected via Object Recognition in Augmented Reality," ECAADE, vol. 1, pp. 415-422, September 2019 [Proceedings 37th Architecture in the age of the 4th revolution, Porto, p. 835, 2019].

[12] X. Li, W. Yi, H. Chi, X. Wang, A. Chan, "A critical review of virtual and augmented reality (VR/AR) application in construction safety," Automation in Construction, vol. 86, pp. 150-162, November 2017.

[13] T. Fukuda, K. Inoue, N. Yabuki, "PhotoAR+DR2016: integrating automatic estimation of green view index and augmented and diminished reality for architectural design simulation," ECAADE, vol. 1, pp. 241-250, September 2017 [Proceedings 35th Sharing of computable knowledge, Rome, p. 769, 2017].

[14] A. Coppens, T. Mens, M. Gallas, "Parametric modelling within immersive environments building a bridge between existing tools and virtual reality headsets" ECAADE, vol. 2, pp. 711-726, September 2018 [Proceedings 35th Computing for a better tomorrow, Łódź, p. 860, 2018].

[15] Y. Fukumoto, T. Yanagimachi, M. Nozawa, Y. Ishida, "Sangaku presented by augmented reality: an educational tool for geometry" Advances in Smart Systems Research, vol. 4, pp. 17-24, 2015.

[16] Z. Chen, C. Liao, C. Chu, "An assembly guidance system of tou kung based on augmented reality," CAADRIA, vol. 1, pp. 349-358, May 2018 [Proceedings 35th Learning, Prototyping and Adapting, Beijing, p. 762, 2018].
[17] U. Abe, K. Hotta, Y. Takami, H. Ikeda, Y. Ikeda, "Digital construction: demonstration of interactive assembly using smart discrete papers with RFID and AR codes," CAADRIA, vol. 1, pp. 75-84, May 2017 [Proceedings 35th Protocols, flows, and glitchers, Suzhou, p. 658, 2017].

[18] A. Bartosh, P. Anzalone, "Experimental applications of virtual reality in design education," ACADIA, vol. 1, pp. 458-467, September 2019 [Proceedings 39th Ubiquity and autonomy, Austin, p. 642, 2019].

[19] G. Goepel, "Augmented construction: impact and opportunity of mixed reality integration in architectural design implementation," ACADIA, vol. 1, pp. 430-437, September 2019 [Proceedings 39th Ubiquity and autonomy, Austin, p. 642, 2019].

[20] S. Chaltiel, M. Bravo, A. Chronis, "Digital fabrication with virtual and augmented reality for monolithic shells" ECAADE, vol. 1, pp. 211218, September 2017 [Proceedings 35th Sharing of computable knowledge, Rome, p. 769, 2017]

[21] G. Betti, G. Aziz, S. Ron, "Pop up factory: collaborative design in mixed reality interactive live installation for the make city festival, 2018 Berlin," ECAADE, vol. 1, pp. 115-124, September 2019 [Proceedings 37th Architecture in the age of the 4th revolution, Porto, p. 835, 2019].

[22] A. Fazel, and A. Izadi, "An interactive augmented reality tool for constructing free-form modular surfaces," Automation in Construction, vol. 85, pp. 135-145, 2018.

[23] G. Jahn, C. Newnham, N. Berg, M. Beanland, "Making in mixed reality: holographic design, fabrication, assembly, and analysis of woven," ACADIA, vol. 1, pp. 88-97, October 2018 [Proceedings 38th Recalibration: on imprecision and infidelity, Mexico City, p. 484, 2018].

[24] J. Forren, M. Ramadan, S. Sarrazin, "Action over form: combining offloom weaving and augmented reality in a non-specification model of design," Artificial Realities, vol. 1, pp. 1-7, October 2019 [Proceedings 1 st Virtual as an aesthetic medium in architecture ideation, Lisbon, p. 450, 2019].

[25] G. Jahn, A. Wit, J. Pazzi, "[Bent] Holographic handcraft in large-scale steam-bent timber structures," ACADIA, vol. 1, pp. 438-447, September 2019 [Proceedings 39th Ubiquity and Autonomy, Austin, p. 642, 2019].

[26] Construção com realidade aumentada. Uberlândia: João Pedro, 2020. Available in: https://youtu.be/cOwg2RhntsQd/

[27] A. Runge, M. Hofer, E. Dittrich, T. Neumuth, R. Haase, M. Strauss, A. Dietz, T. Lüth, G. Strauss, "Manual accuracy in comparison with a miniature master slave device: preclinical evaluation for ear surgery," Studies in health technology and informatics, vol. 163, pp. 524-530, February 2011. 